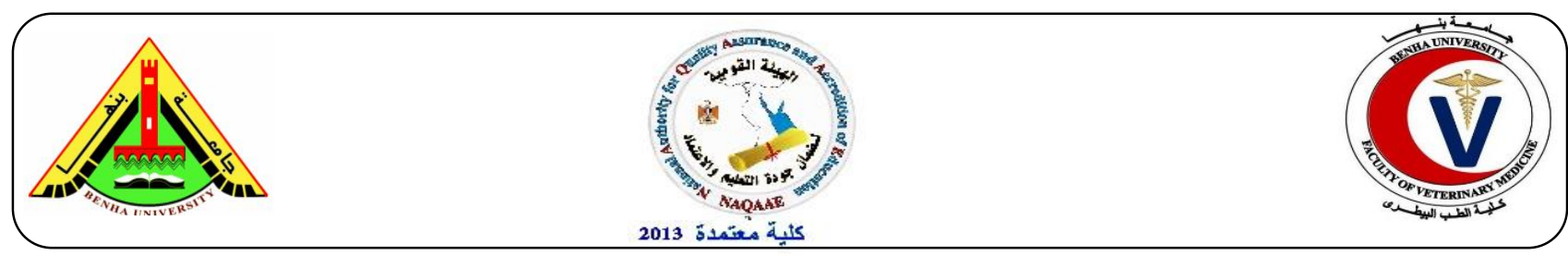

\title{
Enhancement of Immunogenicity and Protective Efficacy against Bovine Respiratory Disease Viruses (BVD, BoHV1, PI3, and BRS) Vaccine.
}

\author{
El-Bagoury, G.F. ${ }^{1}$, Manal. A. Mansour ${ }^{2}$ and Iman. K.A. Kassem ${ }^{2}$ \\ ${ }^{1}$ Department of Virology, Faculty of Veterinary Medicine, Benha University \\ ${ }^{2}$ Veterinary serum and vaccine research institute, Abbassia, Cairo
}

\section{A B S T R A C T}

A highly potent pneumo-4 vaccine of combined inactivated Bovine Viral Diarrhea Virus, Infectious Bovine Rhinotracitis Virus, Parainfluenza-3 Virus and Bovine Respiratory Syncytial Virus could play a valuable role in respiratory disease control program in calves. In this study we determined the influence of virus concentration by polyethylene glycol (PEG) precipitation, and the use of montanide oil \& gel as adjuvants on the immune response of calves to the inactivated Pneumo-4 vaccine. Two batches of concentrated viruses' vaccine were prepared, one adjuvanted with aluminum hydroxide gel and the other adjuvanted with oil. Both batches administered twice to six-month-old seronegative calves through intramuscular (IM) route. Different doses, $2 \mathrm{ml}, 2.5 \mathrm{ml}$, and $3 \mathrm{ml}$ of the concentrated gel adjuvanted \& $2.5 \mathrm{ml}, 3 \mathrm{ml}$ and $3.5 \mathrm{ml}$ of concentrated oil vaccine were tested compared with $5 \mathrm{ml}$ for the commercial un-concentrated Pneumo-4 vaccine. The induced BVDV, IBRV, PI-3V and BRSV antibodies were followed up in the sera of vaccinated calves up to12M post vaccination (M.P.V.) using Serum Neutralization Test (SNT). The result revealed that concentration of viruses by PEG improve the quality of antigen used in the vaccine formulation to produce a good quality combined inactivated vaccine. Also, both concentrated adjuvanted vaccines gave a significant effect on all vaccinated animals but the oil adjuvanted vaccine was particularly better and effective than gel adjuvanted where oil adjuvanted vaccine induced early protective immune response and showed prolongation of antibody secretion in comparison to the Aluminum Hydroxide gel.

Keywords: Inactivated Pneumo-4 vaccine, polyethylene glycol, adjuvants, SNT.

(http://www.bvmj.bu.edu.eg) (BVMJ-36(1): 429-436, 2019)

\section{INTRODUCTION}

Adjuvant plays very important roles in the success of the efficacy of vaccine production. Two main technologies for veterinary adjuvants in the field can fit with multiple expectations as safety \& efficacy in various species such as aluminum salts and emulsions. (Lindbald, 2004 and Oda Kietal, 2006) Oil adjuvants, water-in-oil-in-water (W/O/W), have proved their efficacy in bovine vaccine as they induce rapid and long-term immune response with various antigens. Also, concentration and purification of antigen in vaccine production has many advantages to produce large quantities of product that can be stored in smaller units, also improve immunological activity of viruses and decrease the dose given to the animal (Zeinab and Nehal, 2010). 
Bovine Respiratory Disease (BRD) is a worldwide health concern in stocker and feedlot cattle causing morbidity and mortality in young cattle with major economic losses to the producer. That occurs most often within 4 weeks after weaning. As the weaning process is a stressful time for calves (Windeyeo and Leslie, 2012 and Hilton, 2014). At that time, calves are handled, commingled, and shipped to other locations. During this period, calves may be exposed to many infectious agents that cause BRD (Taylor et al., 2010). BRD is a "multi-factorial syndrome" that is dependent on a number of different causes. A viral infection combines with a bacterial agent, which is often then aggravated by stress. (Kasimanickam, 2010).

The most common viruses involved with BRD include Bovine Viral Diarrhea (BVD), Infectious Bovine Rhinotracheitis (IBR), Bovine Respiratory Syncytial (BRS), and Parainfluenza Type-3 (PI-3V) Viruses. Exposure to these viruses can cause severe damage to the respiratory tract. Inactivated vaccine against these viruses was commonly used for prophylaxis purposes inducing good results of protection (Knezevic et al., 1990 and Hussein et al., 1996).

Induction of protective immunity using inactivated antigens requires their association with effective adjuvants which are selected according to various criteria in order to get the best balance between efficacy and safety.

Our study aimed to use montanide oil \&PEG concentrated antigens to enhance the immunogenicity of vaccine (locally prepared vaccine containing BVD, IBR, PI-3, and BRS viruses) compared with commercial one and elevate the immune response against its components.

\section{Materials and methods}

\subsection{Virus strains and sampling:}

BVD (Egyptian BVDV cytopathic Iman strain of a titer $10^{6.5} \mathrm{TCID}_{50} / \mathrm{ml}$ ), BoHV-1 (A local
Abou Hammad strain of a titer $10^{7.5}$ TCID $50 / \mathrm{ml}$ ), PI-3V (Reference Egyptian strain "strain $45^{\circ}$ of a titer $10^{8} \mathrm{TCID} 50 / \mathrm{ml}$ ), and BRSV (strain $375 \mathrm{~L}$ of titer $10^{6.5} \mathrm{TCID}_{50} / \mathrm{ml}$ ) were passage on MDBK cell line to be ready to prepare viruses. The viruses were collected after freezing and thawing where the supernatants of the viruses were harvested and titrated (Mohanty and Lillie, 1965). Inactivation process was initiated with a final concentration of $0.01 \mathrm{M} \mathrm{BEI}$ at $37^{\circ} \mathrm{C}$ and stopped by addition of cold sodium thiosulphate with a final concentration of $2 \%$. The inactivated viruses were purified and concentrated by $8 \%$ polyethylene glycol (PEG) 6000 (Killington et al., 1996). THE viruses were stirred with PEG at $4^{\circ} \mathrm{C}$ followed by sedimentation of PEG adsorbed viruses' particles by centrifugation at 4500rpm for $30 \mathrm{~min}$ at $4^{\circ} \mathrm{C}$. The particles were eluted from the PEG sediment using tris-buffered saline that was consisted of $8 \mathrm{gm} \mathrm{NaCl}, 0.2 \mathrm{KCl}, 3 \mathrm{gm}$ tris base in liter of DDW, pH 7.4 (Sambrook and Russell, 2001). The viruses' particles were liberated in the buffer and followed by two successive centrifugations of the suspension at 4500rpm for $15 \mathrm{~min}$ at $4^{\circ} \mathrm{C}$. The PEG treated viruses were sterilized by syringe filter.

The concentrated inactivated viruses culture were mixed together and divided into two portions; the aluminum hydroxide gel adjuvant was added as $20 \%$ to one portion and montanide oil (ISA 206) was added V/V to The other portion for preparation two experimental batches of concentrated vaccines and subjected to evaluation using different vaccinal dose ( El-Bagoury et al. 2015 and 2018).

\subsection{Reference hyper-immune sera:}

Reference hyper-immune sera against BVDV, IBRV, PI-3 and,BRSV were obtained from Department of the Rinderpest like diseases, VSVRI, Abbassia, Cairo. It was used in SNT.

\subsection{Calves and experimental design:}

Twenty-four Friesian apparently healthy unvaccinated calves aged 6-9 month and of 
about $150-200 \mathrm{~kg}$ body weight. These calves were sero-negative for BVD, IBR, PI-3 and BRS viruses as screened by SNT. They were allotted into four groups (G1, G2, G3, G4) kept in separate breeding rooms. The divided groups were as follows:

G1: contained 9 calves divided into three subgroups according to the used dose (3 calves/group), each calfe was vaccinated intramuscularly with $(2.0,2.5,3 \mathrm{ml} /$ group $)$ respectively of the concentrated inactivated Pneumo-4 vaccine (adjuvanted with alum hydroxide gel) as two doses 2 weeks apart.

G2: contained 9 calves divided into three subgroups (3 calves /group) vaccinated intramuscularly with $2.5 \mathrm{ml}, 3 \mathrm{ml}$, and $3.5 \mathrm{ml}$ respectively of the concentrated inactivated Pneumo-4 vaccine (adjuvanted with Montanide oil 206) as two doses 2 weeks apart. G3: contained three calves, vaccinated intramuscularly with $5 \mathrm{ml}$ of the commercial inactivated Pneumo-4 vaccine as two doses 2 weeks apart.

G4: the three calves left as non-vaccinated control group.

Serum samples were collected from all calves before vaccination with the first dose of the vaccine and after vaccination at certain time 0day, 2 week, 1 month, and then monthly till the end of experiment. The vaccinated and unvaccinated control calves were observed clinically during the experiment for any clinical abnormalities during the experiment. The body temperature of the calves was recorded daily before and after vaccination for three weeks.

\subsection{Serum Neutralization Test (SNT):}

It was carried out as described by Rossi and Kiessel, (1971). Sera were diluted two-fold dilution in HBSS. Equal amounts of each sera dilution and the virus suspension containing $100 \mathrm{TCID} 50 / \mathrm{ml}$ were added, mixed together and incubated for 60 minutes to interact. $100 \mu \mathrm{l}$ of the mixture was then inoculated into duplicate wells containing confluent monolayer of MDBK cell culture, virus and cell control were included, then incubated at 37o $\mathrm{C}$ for one hour. 100 $\mu 1$ of MEM were added. The plates were incubated at $37^{\circ} \mathrm{C}$ and were examined daily to detect specific CPE. The Serum Neutralizing Antibody titers of the tested serum samples were expressed as $\log 10$ of the reciprocal serum dilution that protects $>50 \%$ of micro titration plate dilution wells, and these following the calculation formula of Reed and Muench, (1938).

\section{RESULTS}

Evaluation of humoral immune response in sera of calves following vaccination with concentrated inactivated Pneumo-4 vaccine adjuvanted with aluminum hydroxide gel; It was observed that, neutralizing antibodies in sera persist at their higher level from the 2 nd W.P.V. (time of 2 nd dose of vaccination) till the $6 \mathrm{~m}$ for all viruses contained in the vaccine as measured by SNT. The groups of calves vaccinated with $2.5 \mathrm{ml}$ or $3 \mathrm{ml}$ mostly gave the same immune response but higher than that given $2 \mathrm{ml}$, table (1). The control nonvaccinated group showed no neutralizing antibody response .

The mean neutralizing antibody in sera of calves vaccinated with oil adjuvant Pneumo-4 vaccine (Group 2) were gradually increased from 14th day post vaccination (D.P.V.) within the minimal protective level for all viruses then reached highest level at 3rd month post vaccination (M.P.V.) and still within the minimal acceptable titer of protective level till the end of the experiment ( 9 months) for all viral components of the prepared vaccine (IBR, BVD, PI-3 and BRS virus). (Table 2).

For calves vaccinated with commercial inactivated Pneumo-4 (G3), valuable neutralizing antibody titers were detected by the 2nd week with lower antibody titer compared with different doses of concentrated vaccine till the end of experiment as shown in Table (3). 
Table1. Mean serum neutralizing antibody titer of BVD, B, PI-3, and BRS viruses in sera of calves vaccinated with different doses of concentrated inactivated Pneumo-4 vaccine adjuvanted with aluminum hydroxide gel.

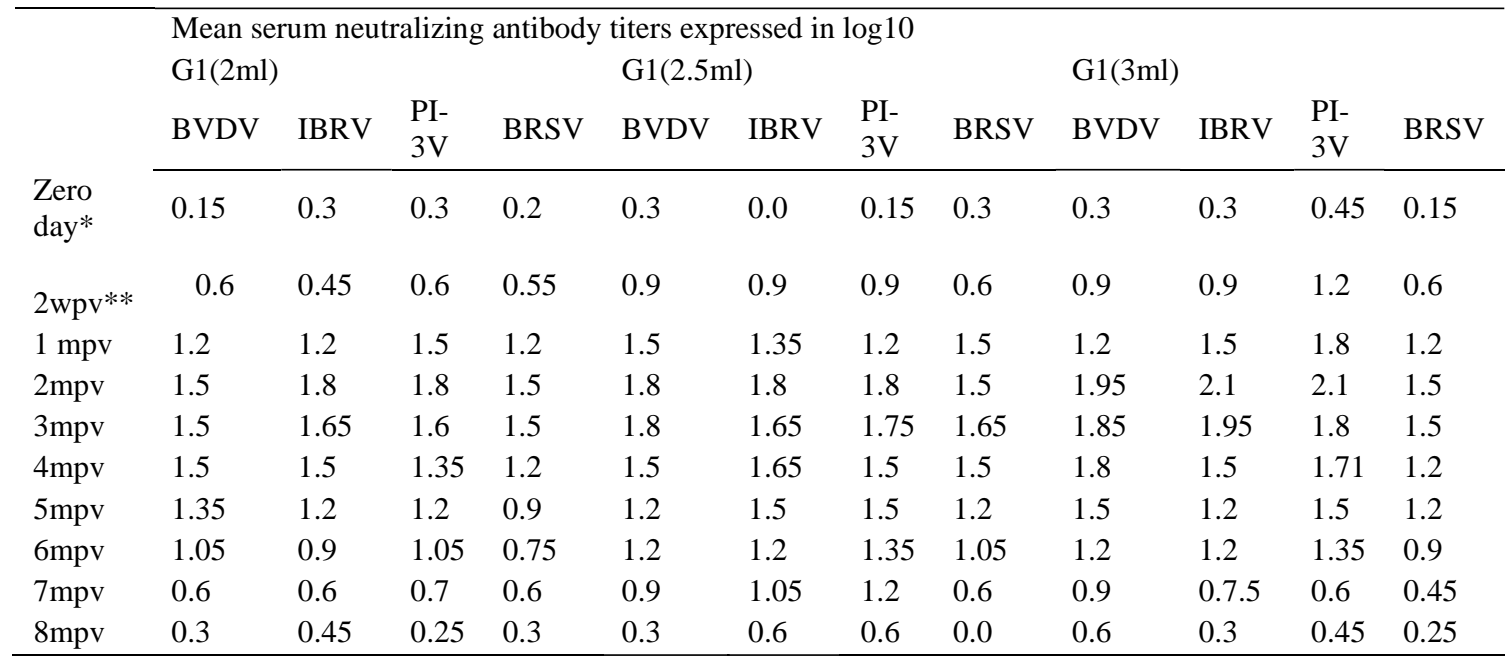

$*$ Time of $1^{\text {st }}$ dose of vaccination, $* *$ time of $2^{\text {nd }}$ dose of vaccination.

Table 2. Mean serum neutralizing antibody titer of BVD, IBR, PI-3, and BRS viruses in sera of calves vaccinated with concentrated inactivated Pneumo-4 vaccine adjuvanted with Montanied oil (ISA 206).

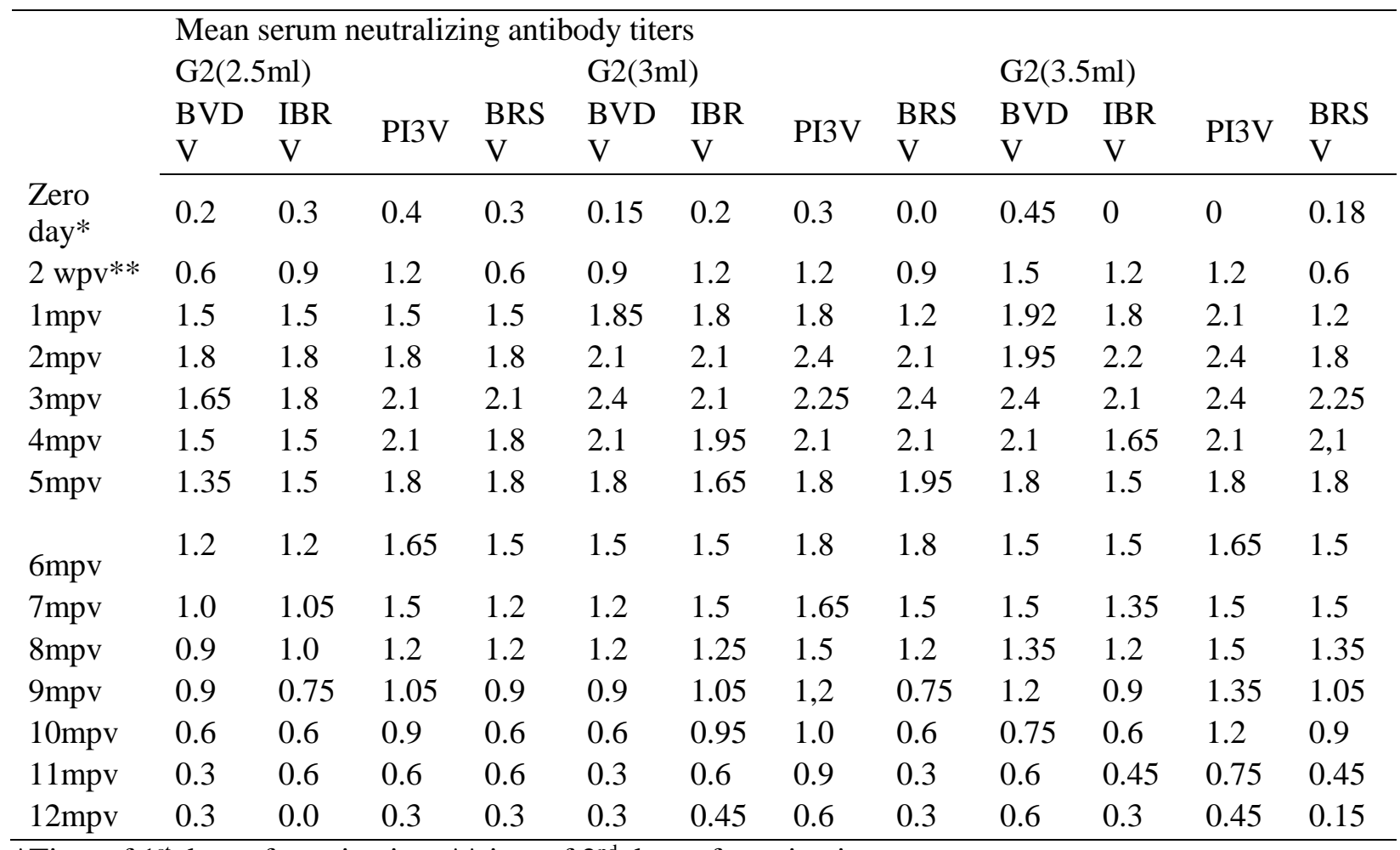

*Time of $1^{\text {st }}$ dose of vaccination, $* *$ time of $2^{\text {nd }}$ dose of vaccination. 
Table 3. Mean neutralizing antibody titer against IBR, BVD, PI-3 and BRS viruses in sera of calves vaccinated with pneumo-4 vaccine adjuvanted with Aluminum hydroxide gel.

\begin{tabular}{|c|c|c|c|c|}
\hline & \multicolumn{4}{|c|}{ Mean serum neutralizing antibody titers } \\
\hline & BVDV1 & IBRV & PI3V & BRSV \\
\hline Zeroday* & 0.3 & 0.24 & 0.3 & 0.21 \\
\hline $2 \mathrm{wpv} * *$ & 0.9 & 0.9 & 0.9 & 0.54 \\
\hline $1 \mathrm{mpv}$ & 1.5 & 1.3 & 1.3 & 1.35 \\
\hline $2 \mathrm{mpv}$ & 1.8 & 1.95 & 2.1 & 1.62 \\
\hline $3 \mathrm{mpv}$ & 1.7 & 1.8 & 1.8 & 1.5 \\
\hline $4 \mathrm{mpv}$ & 1.5 & 1.65 & 1.6 & 1.2 \\
\hline $5 \mathrm{mpv}$ & 1.2 & 1.5 & 1.3 & 1.05 \\
\hline $6 m p v$ & 0.9 & 1.1 & 0.75 & 0.78 \\
\hline $7 \mathrm{mpv}$ & 0.69 & 0.51 & 0.6 & 0.54 \\
\hline $8 \mathrm{mpv}$ & 0.54 & 0.3 & 0.45 & 0.15 \\
\hline
\end{tabular}

*Time of $1^{\text {st }}$ dose of vaccination, $* *$ time of $2^{\text {nd }}$ dose of vaccination, the groups of cattle were vaccinated with $5 \mathrm{ml}$ of commercial inactivated pneumo- 4 . The expected protective titers were 0.9 for BVDV-1 and 0.6 for IBRV, PI-3V and BRSV (Fulton et al, 1995).

\section{DISCUSSION}

The use of inactivated polyvalent vaccines produces good results for protection of calves against BRD with reduction of mortality and decrease incidence of the respiratory disease. The efficacy of these vaccines is variable depending on many factors including animal's age and immune status, virus pathogenicity and its dose and the presence of multiple viral and bacterial infections (Engelken, 1997).

Vaccines can be effective for reducing not only susceptibility but also for reducing shedding of infectious BRD agents to other calves (Frank et al., 2002/2003).

This work was carried out to prepare and evaluate PEG concentrated combined inactivated viruses (Pneumo-4) using aluminum hydroxide gel and montanide oil ISA206 as adjuvants in calves to establish whether a correlation exists between antigen payloads and to examine associated changes in the immune response and estimate the effective dose for controlling of such infections.

There is evidence that increasing the antigen payload promoted a more rapid and greater systemic antibody response as well as impeding local virus replication through antibody mediated protection (Barbett et al., 2004).

Immune stimulation due to the vaccine was measured by the neutralization test since it is the most virus specific serological response and neutralization test is a recognized defense mechanism in many viral infections.

The titer of neutralizing antibodies which was detected in the sera of vaccinated calves is much increased in concentrated Pneumo-4 vaccine by increasing its dose, where calves vaccinated with $2.5 \mathrm{ml}$ or $3 \mathrm{ml}$ mostly gave the same immune response but higher than that given $2 \mathrm{ml}$ (Table 1) as the antigen payload in PEG concentrated Pneumo-4 vaccine enhanced by the increased dose. This result indicated high potency of the concentrated 
vaccine which is adequate to protect susceptible animals from infection.

Estimation of humeral immune response to the inactivated combined vaccine showed that mean antibodies titers were gradually increased from 2 weeks post vaccination and reached to the highest level at 2 M.P.V. and still in a protective level till the end of the experiment for all viruses' components of the vaccine compared to the non-vaccinated group as measured by SNT (Table 1). This agreed with the result which reported that the minimum accepted neutralizing antibody titers were $0.9 \log _{10}$ for BVDV and $0.6 \log _{10}$ for IBRV, PI-3V, and BRSV (Fulton et al., 1995). Duration of immunity elicited by aluminum hydroxide gel vaccine was short-lived and antibody concentration rapidly falls over periods of -6 months after administration. (Ellis et al., 2005, El-Bagoury et al., 2012).

Regarding potency evaluation of the prepared oil adjuvant and commercial vaccines, where immune stimulation due to the vaccine was measured by the neutralization test, the results obtained from Table (2) revealed that all vaccinated calves in Group (2) developed neutralizing antibodies against all viral components of the vaccine showing clearly that Montanide oil prepared vaccine is highly immunogenic and inducing of humoral immune response more than 8 M.P.V.

The previous results revealed that the immune response against the components of the vaccine prepared from PEG concentrated viruses was greater than that prepared from unconcentrated virus. This result agreed with previous work (Lyer et al., 2001) who found that vaccines formulated with virus purified with $8 \%$ PEG were more immunogenic than the vaccines formulated with untreated harvest viruses.

Also, the result revealed that the prepared combined inactivated vaccine adjuvanted with the Montanide oil (ISA206) has been proved greatly effective giving duration of immunity more than 9 M.P.V. compared to that of the commercial and concentrated vaccine adjuvanted with Aluminum Hydroxide gel which gives a duration of up to 6 M.P.V.

\section{Conclusion}

In conclusion, concentration of viruses by PEG improves the quality of antigen used in the vaccine formulation to produce a good quality combined inactivated vaccine. Also, both gel and oil adjuvant give satisfactory results by the using of $2.5 \mathrm{ml}$ dose adjuvanted with gel and $3 \mathrm{ml}$ from adjuvanted with oil that able to induce detectable levels of specific antibodies against all virus component of the vaccine.

\section{REFERENCES}

Barnett, P.V., Keel, P., Reid, S., Armstrong, R.M., Statham, R.J., Voyce, C., Aggarwal, N., Cox, S.J. 2004. Evidence that high potency foot-and-mouth disease vaccine inhibits local virus replication and prevents the 'carrier' state in sheep. Vaccine 22: 1221-1232.

Barnett PV, Cox SJ, Aggarwal N, Gerber H, McCullough KC. 2002. Further studies on the early protective responses of pigs following immunization with high potency foot-and-mouth disease vaccine. Vaccine 20:3197-208.

Callan, R. J. and F. B. Garry. 2002. Biosecurity and bovine respiratory disease. Vet. Clin. North Am. Food Anim. Pract. 18:57-77.

G.F. El-Bagoury, E. M. El-Nahas, Manal.A. Mansour and Iman. K.A. Kassem (2015): Antibody responses by calves after vaccination with commercial and enhanced-potency inactivated pneumo3 vaccines. BVMJ, 28 (1): 247-254.

Garb F. El-Bagoury, Ayman S. El-Habbaa, Effat L. El-Sayed, Manal Abd El-Aziz, 2018. Improving performance of 
Pneumo-4 vaccine using montanide oil. BVMJ, 33 (2): 9-15.

El-Bagoury, G.F., El-Nahas, E.M., AbdElfadiel, M. R., Ghaley, H. M. 2012. Evaluation of maternal antibody in calves borne from cow dam vaccinated with inactivated pnumo-5 vaccine. BVMJ, 23 (2): 9-15

Ellis, j., West, K.H., Waldner, C., Rhodes, C. 2005. Efficacy of saponin-adjuvanted inactivated respiratory syncytial virus vaccine in calves. Cand. Vet. J. 46: 2, 155-162.

Engelken T. J., 1997. Preventative programs for respiratory disease in cow/calf operations. Veterinary Clinics of North America - Food Animal Practice 13, 647-660

Frank, G. H., R. E. Briggs, G. C. Duff, and H. S. Hurd. 2003. Effect of intranasal exposure to eukotoxin-deficient Mannheimia haemolytica at the time of arrival at the feedyard on subsequent isolation of $\mathrm{M}$. haemolytica from nasal secretions of calves. Am. J. Vet. Res. 64:580-585.

Frank, G. H., R. E. Briggs, G. C. Duff, R. W. Loan, and C. W. Purdy. 2002. Effects of vaccination before transit and administration of florfenicol at time of arrival in a feedlot on the health of transported calves and detection of Mannheimia haemolytica in nasal secretions. Am. J. Vet. Res. 63:251-256

Fulton R. W., Confer, A. W., Burge L. J., Perino L. J., d'Offay J. M., Payton M. E. and Mock R. E. 1995. Antibodies responses by cattle after vaccination with commercial viral vaccines containing BHV-1, BVDV, PI3, BRSV immunogens and subsequent revaccination at day 140 . Vaccine, 13: 725-733.

Hilton, W.M. 2014. BRD in 2014. Where have we been, where we are now, and where do we want to go. Anim. Healt Res. Rev. 15(2): 120-2.

Kasimanickam, R. 2010. Bovine Respiratory Disease "Shipping Fever" in Cattle, Washington State University Veterinary Medicine Extension.

Killington, R. A., Stokes, A. \& Hierholzer, J. C. 1996. Virus purification. In Virology Methods Manual, pp. 73-74. Edited by B. W. J. Mahy \& H. O. Kangro. New York: Academic Press.

Knezevic, N.; Kosanovic, P. and Rogan, D. 1990. Immunoprophylaxis of respiratory diseases of cattle with inactivated vaccine. III. Study of the immunogenicity of a bivalent inactivated oil vaccine against IBR and PI3. Veterinarski Glasnik, 44 (7): 503512

Lindbald, E.B. 2004. Aluminum adjuvants in retrospect and prospect - vaccine. Sep 9, 22(27-28): 3658-68.

Lyer A.V; Ghosh S; Singh S.N. and Deshmukh R.A. 2001. Evaluation of three ready to formulate oil adjuvants for FMD vaccine production. Vaccine 191097 1105

Mohanty, S. B. and Lillie, M. G. 1965. A quantitative study of the infectious bovine rhinotracheitis neutralization test.Amer. J. Vet. Res. 26: 892-896.

Oda, K.; Tsukahara, F.; Kutoba, S.; Kida, K.; Kitajima, T.; Hashimoto, S. 2006. Emulsifier content and side effect of oilbased adjuvant in swine - Research in Veterinary science. 81, 51-57. 
Reed, L.T.; Muench, H.A. 1938. Simple method of calculating fifty percent end point. Am. J. Hyg. 27: 493-8.

Rossi, C. R. and Kiessel, G. K. 1971. Microtitre tests for detecting antibody in bovine serum to PI3V, IBRV and BVDV. Microbiol., 22: 32-36.

Sambrook, j. and Russell, D. 2001. Molecular Cloning, A Laboratory Manual, $3{ }^{\text {rd }}$ ed.,3: A1.8.

Taylor, J.D.; Fulton, R.W.; Lehenbauer, T.W.; Step, D.L.; Confer, A.W. 2010. "The epidemiology of bovine respiratory disease: What is the evidence for predisposing factors". The Canadian veterinary journal, La revue veterinaire canadienne, 51 (10): 1095-102.
Windeyeo M.C.; Leslie, K.E. 2012. The effects of viral vaccination of dairy heifer calves on the incidence of respiratory diseases, mortality and growth. Vol. 95, issue 11, pages 6740-6749.

Zeinb, TS. Salama and Nehal S Abdelrahman. 2010. Preparation of inactivated purified concentration canine distemper vaccine. Zag. Vet. j. 40 (2): 95-99. 